\title{
Solid lipid nanoparticles: Influence of composition, fabrication methods and problems, in vitro drug release and intranasal administration provide to access olfactory bulb pathway for SLNs
}

\author{
Anil K. Pawar $1{ }^{*}$, Laxman G. Hatmode ${ }^{2}$, Harshada R. Khandelwal ${ }^{3}$, Vishal C. Gurumukhi ${ }^{4}$ and Shailesh S. \\ Chalikwar 5 \\ ${ }^{1}$ Department of Quality Assurance, R. C. Patel Institute of Pharmaceutical Education and Research, Dist.-Dhule, Shirpur, \\ Maharashtra, India 425405. \\ 2 Department of Quality Assurance, R. C. Patel Institute of Pharmaceutical Education and Research, Dist.-Dhule, Shirpur, \\ Maharashtra, India 425405. \\ ${ }^{3}$ Department of pharmaceutics, R. C. Patel Institute of Pharmaceutical Education and Research, Dist.-Dhule, Shirpur, \\ Maharashtra, India 425405. \\ ${ }^{4}$ Department of Quality Assurance, R. C. Patel Institute of Pharmaceutical Education and Research, Dist.-Dhule, Shirpur, \\ Maharashtra, India 425405. \\ ${ }_{5}$ Department of Quality Assurance, R. C. Patel Institute of Pharmaceutical Education and Research, Dist.-Dhule, Shirpur, \\ Maharashtra, India 425405.
}

GSC Biological and Pharmaceutical Sciences, 2021, 14(02), 126-142

Publication history: Received on 16 January 2021; revised on 17 February 2021; accepted on 19 February 2021

Article DOI: https://doi.org/10.30574/gscbps.2021.14.2.0049

\begin{abstract}
Background: Solid lipid nanoparticles (SLN) have drawn increasing interest in recent years. These nanoparticles are formed from stable or solid lipid mixtures and then stabilized by emulsifiers. As nanoparticles, colloidal particles running in size somewhere in the 10 to $1000 \mathrm{~nm}$ range are known. SLN provides fascinating properties, such as minimal scale, massive surface area, high medication piling, correspondence of stages at the interface, and is interested in their ability to enhance drug execution.
\end{abstract}

Main text: This paper provides a description of the choice of ingredients, the effect of lipids and their structure on the formulation, and the various methods of processing SLN. We explain the characteristics of SLN stability and the possibilities of SLN stabilization by lyophilization in this article. The relation between drug absorption and the complexity of SLN dispersions, which involves the existence of other colloidal structures and the physical state of the lipid, is uncommonly considered. We define the possible problems of SLN preparation and performance on the basis of characterization. First, the nasal route was known to accomplish the avoidance of first-pass hepatic metabolism in order to maximize absolute bioavailability, and secondly, the immediate nose-to-brain pathway to enhance the delivery of brain medicines. SLNs have been designated to increase drug permeability through the blood-brain barrier as a drug delivery device (BBB).

Conclusion: To sum up, this article gives insight SLNs a colloidal drug carrier places together the compensations of polymeric nanoparticles, SLNs have numerous benefits such as easy incorporation of lipid and lipophilic as well as hydrophilic drugs, suitable physical stability, and available at low cost and easy to manufacture. The nasal route was accepted to exploit first its prevention of the hepatic first-pass metabolism to increase the absolute bioavailability, and second, the direct nose-to-brain pathway to enhance the brain drug delivery. SLNs were chosen as a drug delivery system to improve drug permeability across the blood-brain barrier (BBB) and consequently its brain delivery.

\footnotetext{
${ }^{*}$ Corresponding author: Mr. Anil K. Pawar

Department of Quality Assurance, R. C. Patel Institute of Pharmaceutical Education and Research, Dist.-Dhule, Shirpur, Maharashtra, India 425405

Copyright (@ 2021 Author(s) retain the copyright of this article. This article is published under the terms of the Creative Commons Attribution Liscense 4.0.
} 
Keywords: Solid Lipid Nanoparticles; Lipid Nanocarriers: High-Pressure Homogenization (HPH); Lyophilization; Intranasal Delivery; Blood Brain Barrier (BBB)

\section{Graphical abstract:}

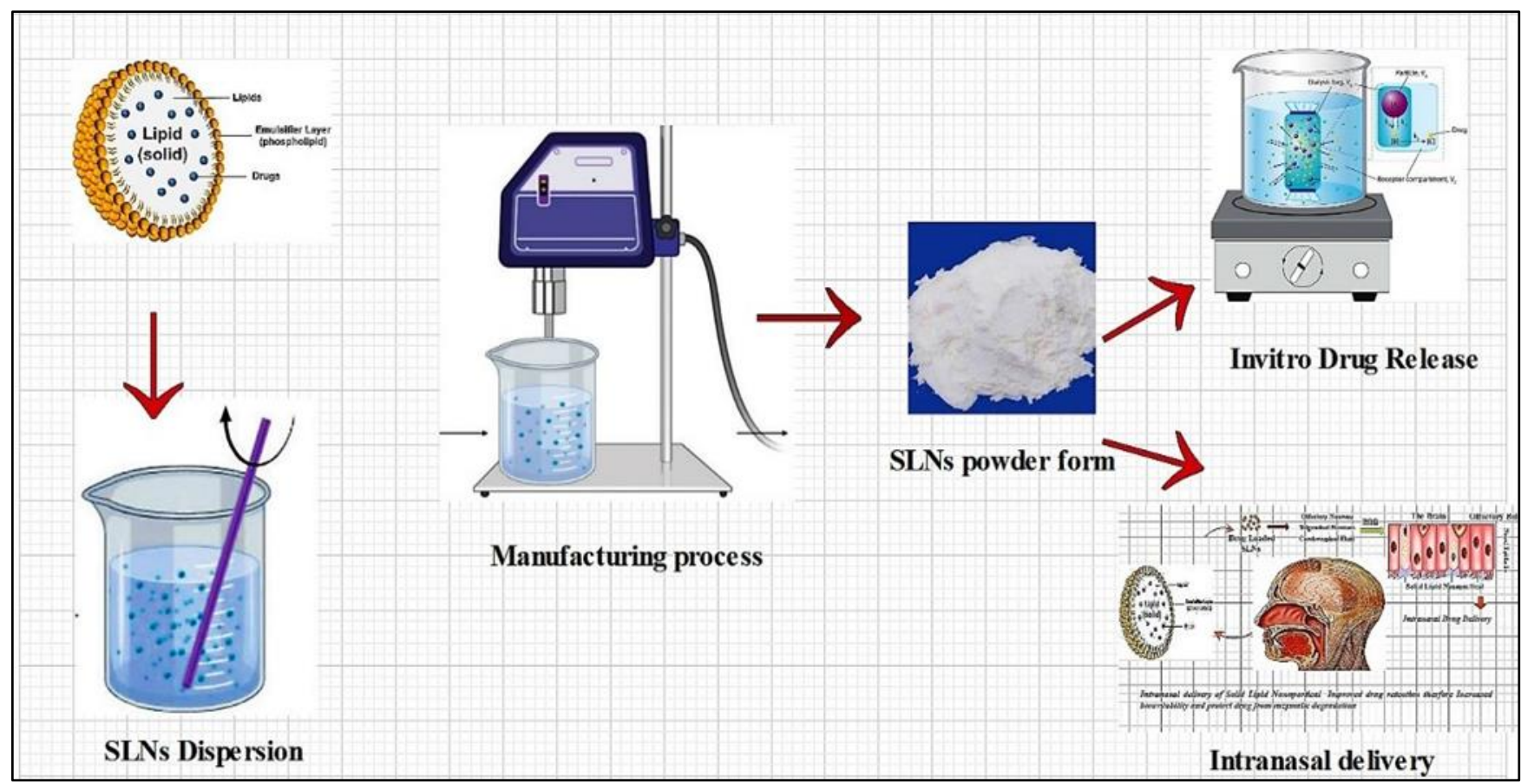

\section{Introduction}

The prefix "nano" comes from the Greek word "Nanos," meaning to predominate. In 1974, the word "Nanotechnology" was written by Prof. Norio Taniguchi at Tokyo Science University. In his book Engines of Creation: The Coming Age of Nanotechnology, Drexler further used this concept in 1986 [1].

A revolution in the sciences has been instigated by rapid advances in the capacity to manufacture nanoparticles of equal scale, form, and structure. In recent years, the production of lipid-based drug carriers has been commonly taken into consideration. SLNs are the cutting edge of nanotechnology's quickly evolving sector with unique expected uses in drug distribution and research, such as in other fluctuating sciences. Lipid nanoparticles deal with the ability to create new therapeutics because of their extraordinary size-dependent properties. An additional paradigm of drug distribution that could be used for the auxiliary and tertiary degree of drug targeting provides the potential to link drugs into nanocarriers. Therefore, powerful lipid nanoparticle holds unbelievable confidence that the target of controlled and site-clear distribution of drugs is achieved and has therefore attracted comprehensive analyst attention.

Over the last 20 years, there has been only one revolutionary carrier system that can be used as a significant creative assurance in the dermal zone, the liposomes that Dior first brought to the cosmetic industry in 1986. Liposomes were sold in drug products for a definite interval several years later. The liposome as a novel carrier found wide consideration among general society aside from mechanical advantages. For the past twenty years, a number of other plan criteria have been used. However, for various reasons, none of them discovered an additional large application and none of them obtained analogous liposomes. In comparison to liposomes and emulsions, solid particles have a few positive benefits, such as defending built-in complex blends against chemical oxidation and modifying the release power of the compound. The advantages of liposomes and emulsions are that they are produced from all nearby endured excipients and can definitely be manufactured on a wide scale, which is important for the industry to be known by a transporter.

The solid polymeric nanoparticles developed by means of non-biodegradable and biodegradable polymers with sizes ranging from 10 to $1000 \mathrm{~nm}$, which are an additional revolutionary parenteral carrier method, arrived at the beginning of the 1990s. Site-particular targeting and controlled release of the combination drugs are positive conditions for these 
particles. Be it as it might, when internalization into cells, the cytotoxicity of the polymers is essential and the variable is often discussed. In addition, the production of polymeric nanoparticles on a wide scale is tricky. This carrier system has not, therefore, been relevant for the pharmaceutical industry so far [2] [3].

Lipid nanoparticles were well-thought out by instructor R. as drug delivery mechanisms from the earliest starting point of the nineteenth century. H. Müller from Germany as well as M. Gascon out of Italy [4] [5]. This nanoparticles are manufactured and stabilized by emulsifiers from a solid or mixture of solid and liquid lipids. Lipids formed in these nanoparticles are biocompatible and, like fatty substances, unsaturated fats, steroids and waxes, are tolerated by the body. What's more, creating a combination of emulsifiers could more easily stabilize the formulations altogether. Lipid nanoparticles have many opinions of consideration in alteration with another particulate system, for sample, the simplicity of largescale production,[6] biocompatible and biodegradable nature of the materials, [7] short toxicity potential, and the possibility of controlled [8] and improved drug release, drug dissolvability development and the chance of both hydrophilic and lipophilic drug combination [9] [10].

Lipidic nanoparticles are not moderately similar to microemulsions, which are solid thermodynamically stable suspensions of surfactant and cosurfactant-stabilized oil and water. Particle size and size distribution, zeta potential, degree of crystallinity, drug loading, entrapment efficacy, and drug release profile are the most relevant factors in the characterization of lipid nanoparticles [11].

Three types of lipid nanoparticles are available: equivalent drug-lipid matrix, drug-enriched core, and drug-enriched shell. Drug release as lipid nanoparticles in matrix formulation is largely dependent on the type of matrix and site of the drug; for example, drug release from the nanocarriers indicates extra sustained release profile in the third shape [12]. Lipid matrix synthesis, surfactant concentration and manufacturing conditions may also affect drug release profiles for sample, temperature and blending volume. The ease of large-scale processing and its low toxicity potential are perhaps the most important reasons for using lipid nanoparticles as a viable choice for previous polymeric nanoparticles [13].

Solid lipid nanoparticles (SLNs) are receiving a lot of consideration as potential colloidal drug carriers these days. Nanostructured lipid carriers (NLCs) have been developed to address the drawbacks of SLNs, such as capacity drug leakage and deficient total drug load [14]. NLCs are considered as the second generation. A mixture of solid lipids with incompatible liquid lipids is based on NLCs [15]. NLC exhibits various characteristics for medicinal use, e.g. g. Regulated release of active compounds, drug targeting, and enhancement of drug penetration measurement into the mucosa. For a few routes of administration, SLN and NLC were investigated, for example, parenteral oral and productive route giving controlled distribution systems to multiple active routes [16]. In pharmaceutical, cosmetics and dermatological info, SLN and NLC were used. SLN and NLC both have a lot of highlights that are invaluable to the delivery system of drugs [17].

\section{Solid lipid nanoparticles (SLNs):}

Solid lipid nanoparticles (SLNs) are presented as a carrier device for inadequately dissolvable water medicine and special remedial medicine. As nanoparticles, colloidal particles running in size somewhere in the 10 to $1000 \mathrm{~nm}$ range are known. They are integrated from characteristic polymers developed and are ideal for advancing medication transport and reducing lethality [18]. As a drug carrier, they have been developed as an adaptable substitute for liposomes. They are made from manufactured/characteristic polymers and are best suited for enhancing sedate movement and reducing lethality [19]. For example, SLN provides fascinating properties, such as minimal scale, massive surface area, high medication piling, correspondence of stages at the interface, and is interested in their ability to enhance drug execution [20].

Aqueous colloidal dispersions, whose network comprises solid biodegradable lipids, are SLNs. For eg, physical stabilization, an assurance of fused labile drugs from safety, debasement, managed release, and incredible tolerability, SLNs incorporate the great conditions and sustain a key good way from the downsides of a few colloidal carriers of their group. Information of SLN have been developed for various application progressions such as parenteral, oral, dermal, visual, pulmonary, and rectal and have been characterized in vitro and in vivo altogether [10].

For drug targeting, SLNs are known as an attractive colloidal drug carrier device. SLN may also be selected for being exceptionally stable, clean, over polymeric nanoparticles. In nano calculation, these are spherical stable lipid particles spread in an aqueous surfactant arrangement [21]. SLNs consist primarily of a solid hydrophobic core with a monolayer of phospholipid coating. The solid core may involve the drug dissolved or dispersed with the hydrophobic finish of the phospholipid chains implanted in the fat matrix shown in Figure 1 in the solid high melting fat matrix. In this manner, lipophilic or hydrophilic drugs can be produced and appear to meet the requirements for the optimum particulate 
carrier system [22][23]. SLN proposal an enormous improvement in drug delivery when they directed through effective, oral, and parenteral courses. In particular, because of their low aqueous solubility, encapsulation of drugs in SLN can help to solve problems, protect the encapsulated drug from degradation, controlled release is possible, able to cross various biological barriers, and the trapped product assigned to a particular target.

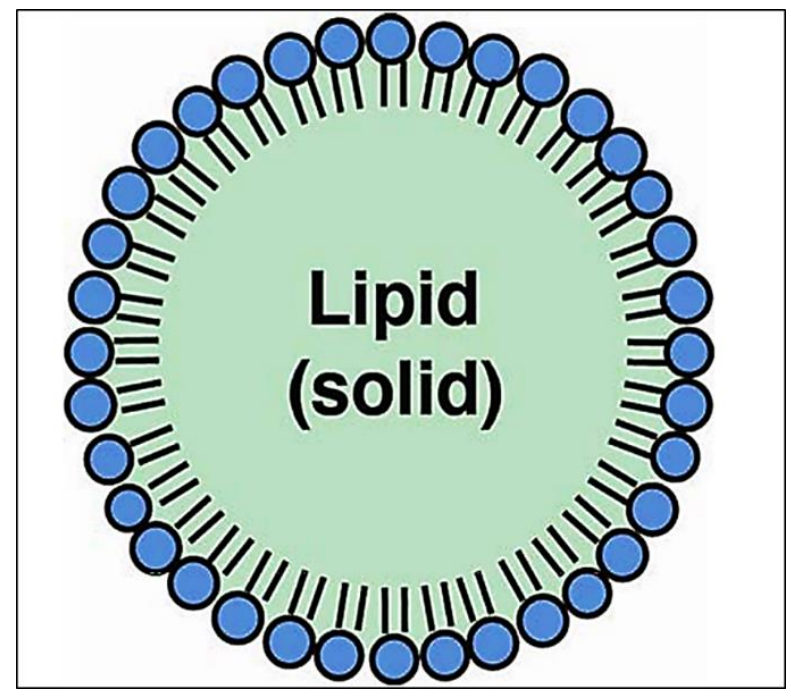

Figure 1 Structure of Solid Lipid Nanoparticle (SLNs)

A noteworthy consideration has been paid on SLN to build up as a drug carrier to target or cross the BBB, as they offer a few preferences furthermore,[24] specifically: Displayed great biocompatibility as existence included of physiological lipids. SLNs offer high medication payload, avoidance of natural solvents during manufacturing. Large-scale production and chances of managed drug delivery may be successfully sterilized. As SLNs deal with increased stability for up to 3 years, an incredibly enormous advantageous role over the other colloidal carrier system [6].

\section{Composition of SLNs}

SLN's structure contains stable lipids, emulsifiers, water and active substances. Table 1 lists the ingredients which are mostly used in the preparation of SLNs.

Table 1 Commonly Used Lipids and Surfactants for the Preparation of SLNs

\begin{tabular}{|l|l|}
\hline Ingredients & \multicolumn{1}{c|}{ Examples } \\
\hline Lipids & $\begin{array}{l}\text { Hard fat sorts: Witepsol W, H, and E, Palmitic acid, Decanoic acid, Stearic acid etc. } \\
\text { Triglycerides: Tricaprin, Trilaurin, Trimyristin, Tripalmitin etc. } \\
\text { Diglycerides: Glyceryl palmitostearate, Glyceryl behenate, Precirol AT0 5 etc. } \\
\text { Monoglycerides: Glyceryl monostearate, Glyceryl behenate etc. } \\
\text { Waxes: Cetyl palmitate, Carnauba wax, Beeswax etc. }\end{array}$ \\
\hline $\begin{array}{l}\text { Emulsifiers and } \\
\text { co- emulsifiers }\end{array}$ & $\begin{array}{l}\text { Ionic surfactants: Sodium cholate, Sodium glycocholate, Sodium taurocholate, Sodium } \\
\text { taurodeoxycholate etc. } \\
\text { Amphoteric surfactants: Egg phosphatidylcholine, Soy phosphatidylcholine, Hydrogenated } \\
\text { soy phosphatidylcholine, Egg phospholipid, Soy phospholipid etc } \\
\text { Non-ionic surfactants: Poloxamer- 188, 407, Polysorbate (20, 60, 80), Span (20 \& 80), etc }\end{array}$ \\
\hline
\end{tabular}

\subsection{Lipids for the manufacturing of SLNs}

A clear advantage of SLNs is that the lipid matrix is made from physiological lipids which decrease the danger of acute and chronic toxicity. Lipids used for the preparation of SLNs include triglycerides, partial glycerides 18, fatty acids, 
steroids and waxes. Most of these lipids except Cetyl palmitate are approved as generally-recognized -as safe. The lipid is the major element of SLNs that affect their particle size, drug encapsulation efficiency, stability and the release profile of the formulations. Selection of suitable lipids for the preparation of SLN is vital, although there are no specific guidelines. Lipids are designated on basis of their capability to solubilize the drug [25]. The solubility of drug can be simply quantified using UV-Visible spectroscopy [26].

\section{Influence of the lipid:}

It has been stated that throughout hot homogenization, the particle size of SLN dispersions growths with upper melting lipids due to higher viscosity of the dispersed phase [27]. In a study, SLN was prepared using fatty acid and lipids. The particle size of SLNs prepared by stearic acid was extra than other lipids as this lipid exhibited some emulsifying property while fatty acid did not show; for this reason, SLNs prepared by stearic acid are better than other lipids [28]. Though, other critical parameters for SLNs formation are dissimilar for different lipids. Samples contain the lipid hydrophilicity, the velocity of lipid crystallization. In all-purpose, increasing the lipid content done 5-10\% in most cases results in larger particles and wider particle size distributions [29]. Degree of crystallinity should also be taken into consideration throughout the collection of lipids for preparation of SLNs. Throughout storage, changes of the crystal lattice might occur in goodwill of thermodynamically stable patterns and this is connected with expulsion of the drug molecules. The lipid modification during storage be contingent upon factors such as miscibility, the solubility of the drug in lipid, the physical and chemical structure of the lipid and polymorphic state of lipid materials. Lipid alteration is a main parameter that regulates the performance of the SLNs to a large extent, because this parameter triggers drug incorporation, release and stability. Various lipids used for the preparation of SLNs are fatty acids, Monoglycerides, diglycerides and triglycerides and individually lipid varies in their chemical, physical. Due to these differences, it is predictable that SLNs prepared by these lipids will be differ to each other in respect of entrapment efficiency (EE), in vitro release and stability.

In general, cumulative the lipid content, EE also escalates up to the certain limit. This might be due to upper content of lipid which offers extra space to entrap the drug and also reduce the escape of drug into the external phase. The presence of long-chain fatty alcohol could lead to the creation of a less ordered solid lipid matrix and leaves enough space to accommodate drug molecules. Among GB and GM, GB showed better EE due to the carbon chain length (lipophilicity) [30].

\subsection{Emulsifiers and co- emulsifiers}

All classes of emulsifiers (concerning charge and molecular weight) are utilized to stabilize the lipid dispersion. Emulsifiers may be used as single or in combination. It has been detected by different researchers that the mixture of emulsifier's strength prevent particle accumulation extra capably than single emulsifier. Emulsifiers used for the manufacturing of SLNs play two quite distinct and important roles, emulsifiers disperse the lipid melt in the aqueous phase throughout the production process, and surfactants stabilize the SLNs in dispersions afterward cooling. Choice of emulsifiers depends on the following factors: a) route of administration. b) HLB value c) Effect of surfactant on particle size d) Role in vivo degradation of the lipid. Non-ionic emulsifiers are favoured for parenteral, oral and nose to brain drug delivery systems as they are less toxic. Among numerous types of emulsifiers, the cationic emulsifiers are more toxic than others [31].

\section{Influence of the emulsifier}

The sort and concentration of emulsifier have a straight influence on the quality of the SLN dispersion. The extraordinary concentration of the emulsifier decreases the interfacial surface tension and eases the particle partition throughout homogenization. Decrease in particle size is allied with an extraordinary rise in surface area during homogenization. The development of primary attention of the novel surfaces competes with the agglomeration of uncovered lipid surfaces. The primary dispersion must have excessive emulsifier molecules, which will promptly cover the new surfaces. Surfactants owning HLB value less than 12 are commonly used to stabilize the SLN dispersion. It is reported that SLN stabilized with surfactant combinations have lesser particle sizes and higher storage stability than a single [32].

The extraordinary concentration of surfactant is not continuously desirable as it leads the bridging between the primary nanoparticles and henceforth accumulative the particle size. Additional, the advanced surfactant concentration may decrease the EE and toxicity related with a surfactant may occur, consequently a high concentration of surfactant should be avoided. Reduction in EE with surfactant at a constant amount of lipid is due to the formation of micellar solutions of a drug resulting in an increase in the solubility of the drug in the water phase [33]. 


\section{Drug Incorporation Models in SLNs}

Part of the drug inside the lipid nanoparticles depend on preparation technique, drug lipid solvency and type of surfactant and its concentration. There are some chiefly three models depicting the incorporation of medication into SLNs as shown in Figure 2 [34] [35].

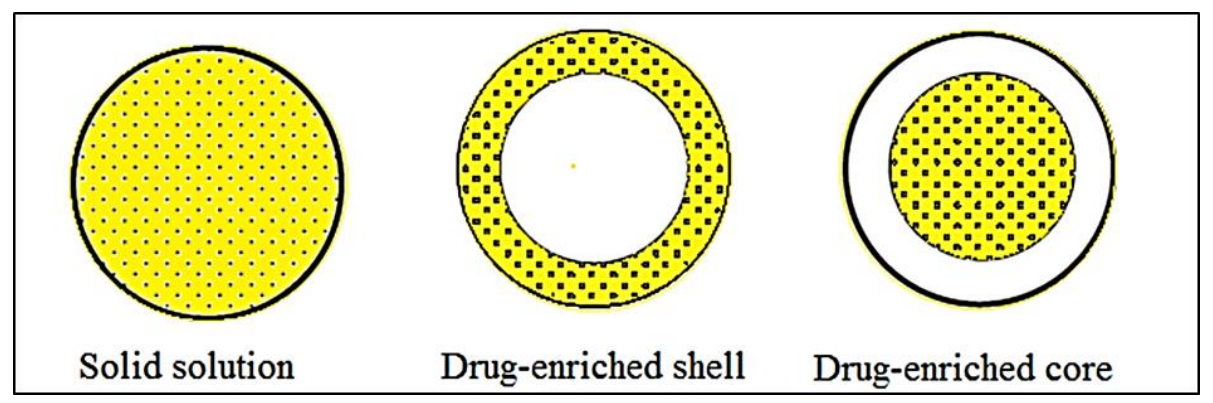

Figure 2 Drug Incorporation Models

\subsection{Core-shell model, drug-enriched core}

The medication might be found mostly in the core of the particles. A medication improved centre is found on the off chance that drug precipitates first before lipid recrystallizes. It is valuable to produce a membrane controlled delivery pattern.

\subsection{Core-shell model, drug-enriched shell}

The medication might be situated in the shell. In an improved shell model, a medication specifically situates at the interface, either by fast solidification of the network lipid or by successful competition of the medication for the interface.

\subsection{Solid solution model}

A medication might be atomically dispersed all through the matrix and present in the undefined structure. For this situation, SLNs are created by cold homogenization and utilizing no surfactant.

\section{Methods of Preparation}

Till now, various techniques have been accounted for the preparation of SLN by various researchers. Not many of them are high-pressure homogenization (hot and cold), Microemulsion, Solvent emulsification (diffusion or evaporation), Solvent injection, Homogenization by sonication, supercritical gas micro- atomization procedure, Liquid flow-focusing and gas displacing technique in microchannels and so on. Among the unique techniques, high-pressure homogenization and microemulsion are the most flexible strategies utilized for SLNs preparation. Some major strategies are talked about as follows:

\subsection{High-pressure homogenization (HPH)}

As a safe technique for SLN preparation, high-pressure homogenization (HPH) is used. Homogenizers of various sizes are produced by small suppliers at a fair cost. At elevated shear pressure, the particles of the submicron go are obtained, which is more, aviation compulsion. Nanoemulsions are produced by HPH for parenteral nourishment. HPH moves the fluid into a limited space at high weights (100 to 2,000 bar) (range of few microns). Over a quick high-speed separation, the liquid changes faster with homogenization, even the high lipid content could be transformed into Nano dispersions. The techniques for SLN assembling are hot and cold homogenization processes. In all cases, a preliminary advance includes that. The lipid matrix used in this step is isolated from the physiological lipids, decreasing the likelihood of chronic and acute toxicity [36]. 


\subsubsection{Hot homogenization technique:}

As per this method, lipids are liquefied by $5-10^{\circ} \mathrm{C}$ warming, dispersed in dissolved lipids over their liquefying stage and drugs. The drug-loaded lipid is dispersed in a hot watery surfactant solution for a pre-emulsion structure that is thus homogenized before the correct size is achieved [37][38].

\subsubsection{Cold homogenization technique}

Drugs are dispersed in melted lipids followed by quick cooling to form a stable solution, as demonstrated by this method. The solid solution is then ground to miniature particles that are homogenized within the sight of the surfactant along these lines at room temperature. The thermolabile drug is reasonable for this technique [39] [40]. The previous three problems related to hot homogenization are overcome by cold homogenization: drug degradation due to temperature, drug circulation during homogenization into the aqueous stage, the difficulty of the crystallization process that prompts a few adjustments and supercooled melt.

\subsection{High shear homogenization}

High shear homogenization and ultrasound are methods of dispersion that were originally used to build solid lipid Nano dispersions. The two techniques are broad and easy to manage. In any case, the existence of microparticles often undermines the dispersion quality. Besides, metal contamination must be thought of if ultrasound is utilized as in Figure $3[41]$.

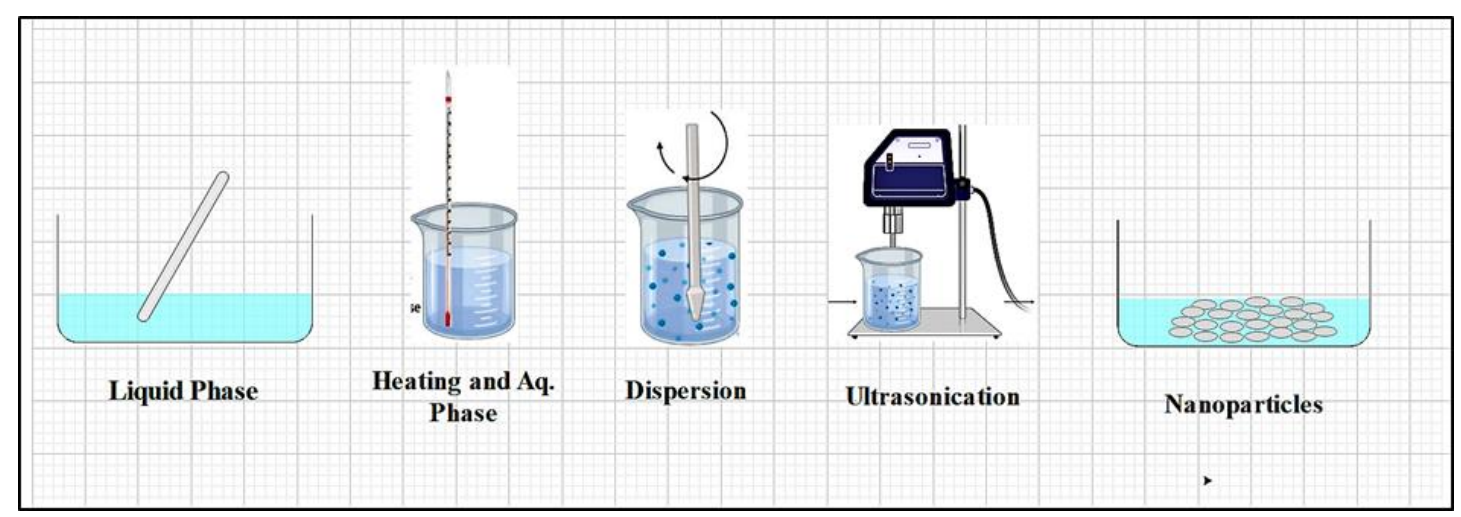

Figure 3 High shear homogenization or ultra-sonication technique

Ahlin et al. utilized a Lak Tek rotor-stator homogenizer to create SLN by melt-emulsification. They examined the impact of various cycle boundaries, counting emulsification time, mixing rate and cooling conditions on the molecule size and the zeta potential. Lipids utilized in this investigation incorporate Trimyristin (Dynasan®114), Tripalmitin (Dynasan $® 116$ ), Tristearin (Dynasan $® 118$ ), a blend of mono-, di-and fatty oils (Witepsol@ W35, Witepsol@H35) and glycerol behenate (Compritol®888 AT0), poloxamer 188 was utilized as a steric stabilizer (0.5 w \%). For Witepsol@ W35 dispersions the accompanying parameters were found to deliver the best SLN quality: mixing for 8 min at 20000 $\mathrm{rpm}$, the ideal cooling conditions: $10 \mathrm{~min}$ at $5000 \mathrm{rpm}$ at room temperature. Conversely, the best conditions for Dynasan ${ }^{\circledR} 116$ dispersions were 10 -min emulsification at $25000 \mathrm{rpm}$ and $5 \mathrm{~min}$ of cooling at $5000 \mathrm{rpm}$ in cool water. Higher mixing rates didn't altogether change the molecule size, yet somewhat improved the polydispersity index. No broad guideline can be gotten from contrasts in the set up ideal emulsification and cooling conditions. As a rule, normal molecule sizes in the scope of 100-200 $\mathrm{nm}$ were acquired in this examination [42].

\subsection{Microemulsion technique}

Gasco and associates developed SLN agreements focused on decreasing the concentration of microemulsions. Biphasic units composed of external and internal mediums are these microemulsions. The blend consists of unsaturated low freezing, an, co, and water. The hot microemulsion is distributed in the cold water [10]. If the microemulsion combination is assumed, the dilution phase can be fixed in Figure 4. There is no extra energy expended in this process to obtain the submicron size. The key steps for the processing of nanoparticles are that they should be carried with simple solvents that easily fit into the fluid level, but more lipophilic solvents are used to achieve large particle sizes [43] [44]. 


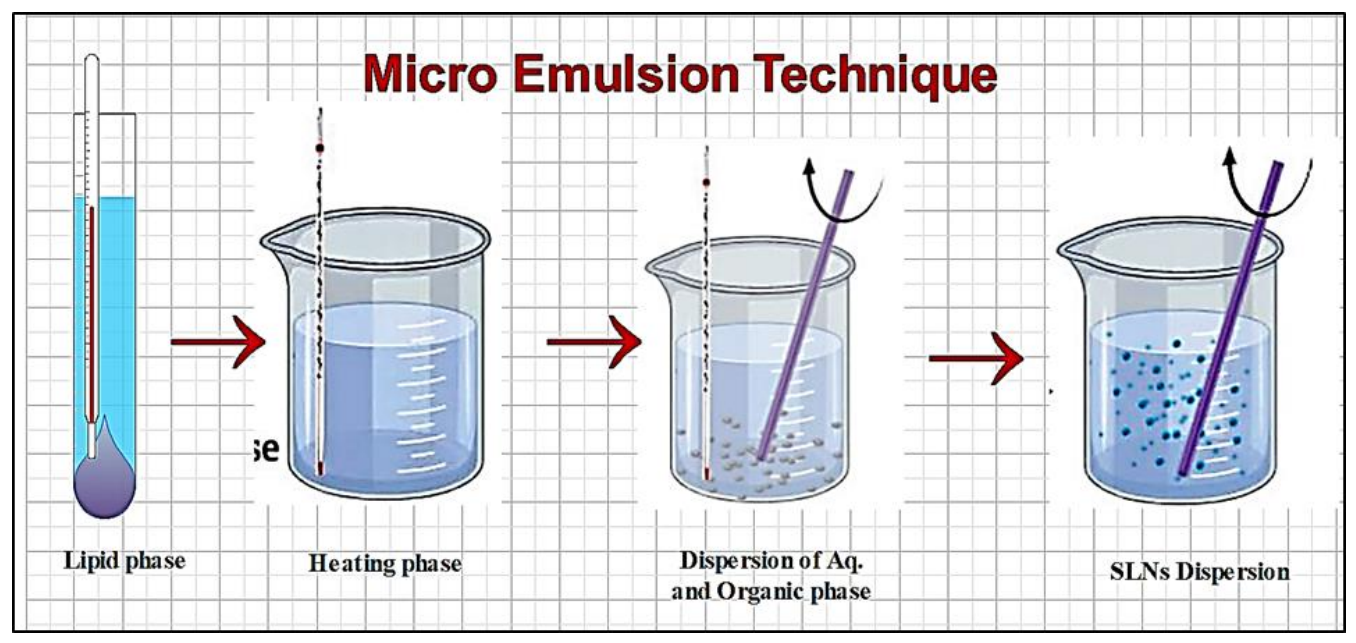

Figure 4 Microemulsion technique

\subsection{SLN prepared by solvent emulsification/evaporation}

The lipophilic substance is dissolved in a normal dissolvable water-immiscible, and is emulsified in an aqueous phase. A nanoparticle dispersion is formed by precipitation of the lipid in the fluid medium upon evaporation of the dissolvable. The mean calculation of the particles obtained was $25 \mathrm{~nm}$ with derivation of cholesterol acetic acid as a model drug and using a combination of lecithin/sodium glycocholate as an emulsifier [45]. The nanoparticles of the cholesterol acetic acid derivation had a mean molecule size of $29 \mathrm{~nm}$. By dissolving the fatty substance in the chloroform, the Tripalmitin nanoparticles. In figure 5 , this structure was emulsified by HPH in a fluid stage.

The organic solvent was extracted by disappearing under decreased tension from the emulsion. The mean particle size, depending on the co-surfactant combination, varies from around 30 to $100 \mathrm{~nm}$. Using bile salts as co-surfactants, particles with normal measurements of as little as $30 \mathrm{~nm}$ were obtained. Comparable small particle size dispersions by dissolving emulsification in comparable arrangements are not feasible. The mean size of particles depends on lipid convergence in the organic process. Very small particles with low-fat organic solvent-related loads must be collected. The efficacy of homogenization decreases with the expanding lipid content due to the higher viscosity of the dispersed process. The advantage of this approach over the previously depicted cold homogenization measure is the shirking of some thermal tension. The use of organic solvents is a distinct drawback [46] [47].

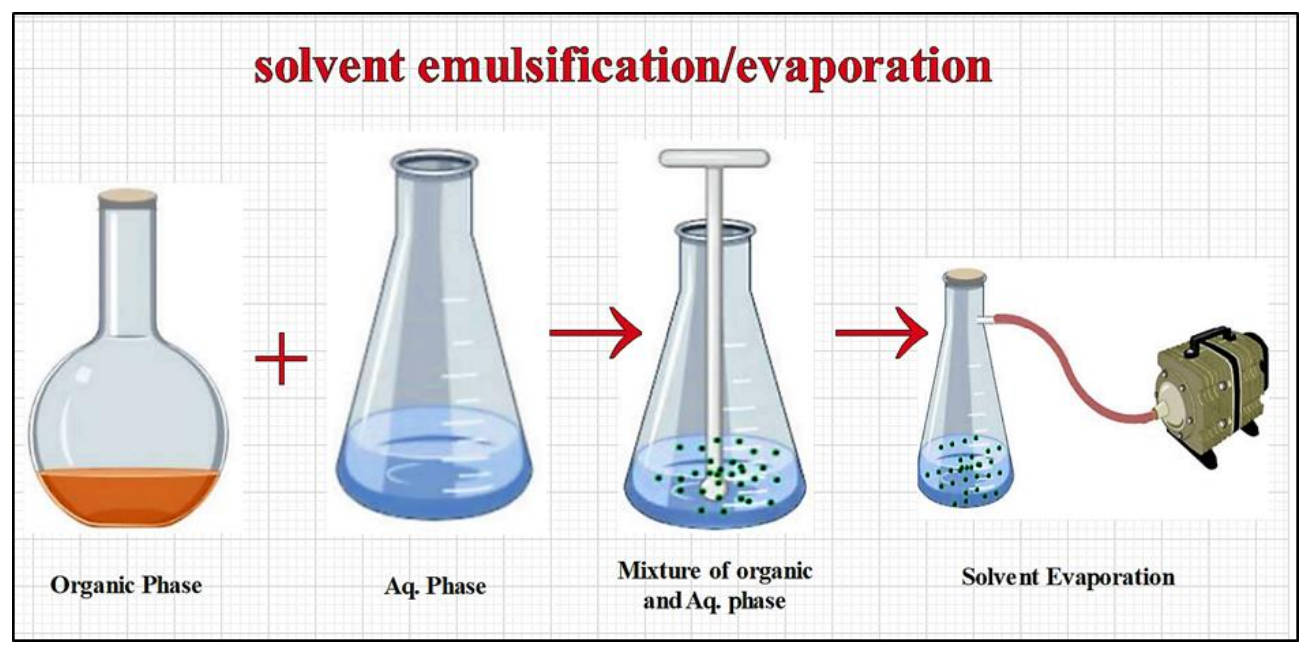

Figure 5 SLN prepared by solvent emulsification/evaporation

\subsection{Solvent emulsification-diffusion technique}

The solvent emulsification-diffusion technique was introduced by Trotta et al (2003). A solvent-in-water emulsion is prepared according to this process, with a partial water-miscible solvent comprising the lipid. It is possible to use lowtoxic, water-miscible solvents, such as benzyl alcohol or butyl lactate. Droplets of the dispersed phase become solidified 
in the form of lipid nanoparticles due to organic solvent diffusion after transferring a transient o/w emulsion into the water followed by continuous stirring. Ultra-filtration will further purify the suspension, and the relation between solvent diffusion and the method of evaporation is defined in Figure 6 [48].

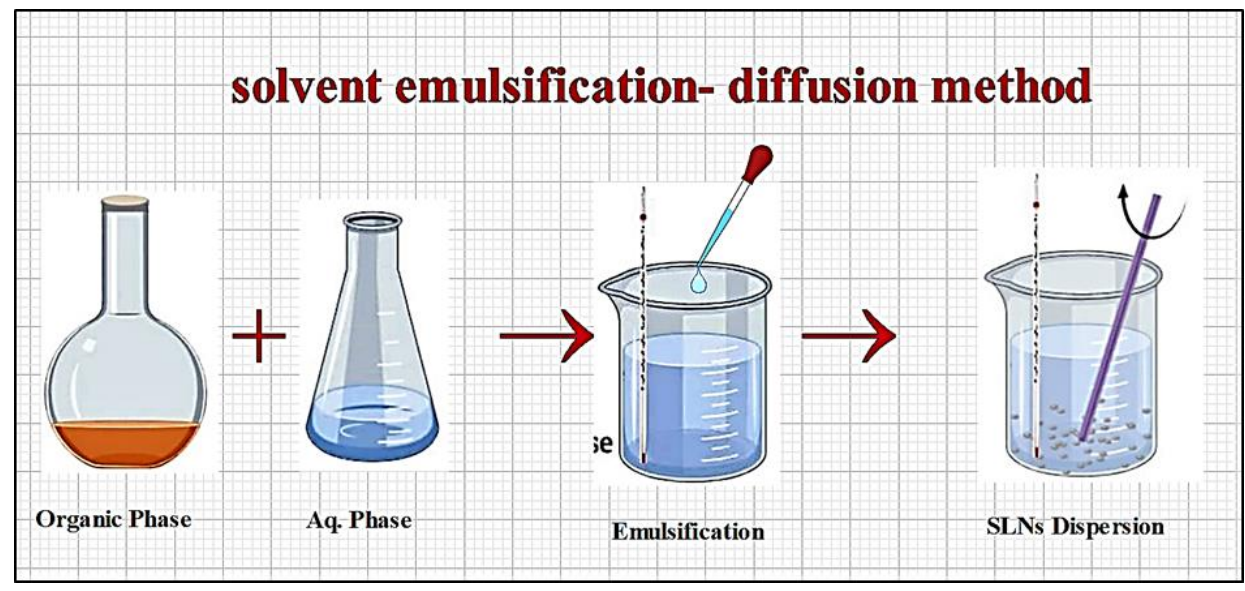

Figure 6 Solvent emulsification- diffusion method

\section{Lyophilisation of SLNs}

The method of transforming the dispersion of aqueous lipid nanoparticles into stable lipid nanoparticle powder is known as lyophilization. Moving to solid form inhibits growth and development of Ostwald and avoids reactions of hydrolysis. Lyophilization can extend the physical and chemical stability of SLNs. It has also been found that the power of the lyophilized SLN powder is greater than that of the dispersion of the SLN. It also includes head possibilities for the inclusion of SLN in pellets, tablets or containers [49].

Capacity stabilization involves chemical and physical points of view and includes stopping degradation reactions and maintaining the original particle sizes. This needs sufficient compound reliability of the SLN ingredients and particles should have a very small distribution of size to keep away from crystal growth because of Ostwald ripening. The formulation should be resistant to variations in temperature which can occur during transport. The particle size of aqueous SLN dispersions has been indicated to be constant for more than 12-36 months. However, this consistency is anything but a general highlight in SLN dispersions and an expansion of particle size can be observed in a shorter timeframe in a significant portion of the situations [50]. Two additional changes between the formulas, however, are required, which may be the cause of additional stability problems.

\subsection{First transformation}

The transition from the aqueous level to the form of powder. There could be a stability issue due to the freezing out effect.

\subsection{Second transformation}

Freeze-dried substance resolubilization, which favours particle aggregation.

The defensive effect of the surfactant may be traded off during lyophilization. It has been found that the rise in the molecule can be avoided by using lipid that does not exceed 5 percent. Issues occur when lyophilization can be overwhelmed by the development of cryoprotective operators known as cryoprotectors [51]. Sorbitol, mannose, maltose, trehalose, glucose and Polyvinylpyrollidone are common cryoprotective specialists used (it focuses somewhere in the range of 10 and 15 percent). They reduce the osmotic activity of water and prefer the solidified example's smooth condition [52] [53].

Cryoprotectors are role holders that avoid interaction with distinct lipid nanoparticles and interface with the surfactants' polar head array and fill in as a kind of pseudo hydration shell. The time of its expansion and grouping of lipids also influences the nature of the prepared mixture, considering the concentration of cryoprotectors. Furthermore, the best results were obtained when the cryoprotectors were applied to the preceding homogenization example. Samples with a lipid content below 10\% displayed less collection than higher-concentration samples. The expulsion of 
water occurs due to lyophilization, resulting in a difference in the properties of the surfactant substrate and thus higher concentration of particle that favour particle aggregation.

\section{Possible problems in SLN preparation and SLN performance}

In comparison to various schemes, SLN gives a few desirable conditions (simple scaling up, shirking of natural solvents, and high content of nanoparticles). In an array of papers, these preferences have been discussed. In any case, the detailed and appropriate investigation of the impediments of these transporter systems has received less consideration. These methods would also be explored in the following part of the article Focuses to consider include drug degradation caused by high pressure, the coexistence of multiple lipid modifications and separate colloidal organisms, the ability of drug loading and the processed kinetics of dissemination.

\subsection{High pressure-induced drug degradation}

It appeared to diminish the molecular weight of polymers [54]. The significant cause was assumed to be high shear pressure and signs of free radical development was identified. Furthermore, this analysis showed that cavitation is less necessary for the polymer degradation component. Without significant improvements in homogenization abilities, cavitation can be suppressed by the application of backpressure. For predicting drug decay, the molecular weight and the general molecular structure are the most important limits Compounds with high molecular weight and long-chain particles are more susceptible than compounds with low molecular weight or circular-shape particles. For example, it was found that HPH causes DNA and egg white corruption [55]. Impact of HPH on peptide lysozyme action by methods of electrophoresis of sodium dodecyl sulphate polyacrylamide gel and rate of lysis of Micrococcus lysolideikticus [56] .

During cold homogenization, the peptide remained in its active state. It can be expressed very well, as per the details in the writing, that HPH-induced drug degradation for most of the medications would not be a real problem. Nonetheless, HPH may not be suitable for the treatment of shear-sensitive compounds [57].

\subsection{Lipid crystallization and drug incorporation}

For the presentation of the SLN transporters, lipid crystallization is a major point. For quite a long time, the relation between lipid shift and drug incorporation has been examined. There is a well-known characterization of lipid modifications. Strategies are largely dependent on measurements of the X-beam and DSC. In any case, the remainder of the details was derived from bulk lipid inquiries. The actions of SLN could differ considerably because of the minuscule size of the particles, what's more, the high measure of surfactant particles that are critical for settling the colloidal lipid dispersion. Surface-related phenomena and lipid-surfactant interactions will also contribute to the lipid molecule's properties to an incredible degree. In the topic of drug integration into SLN, the following four main issues should be considered: The existence of supercooled melts, Lipid modifications, Particle shape, Gelation phenomena [58].

\subsubsection{Supercooled melts}

In the SLN system, this is not odd. It describes the phenomenon that lipid crystallization may not occur even if the example is placed below the lipid melting point at a temperature. Supercooled melts are not Nano suspensions of lipids, but emulsions rather. Supercooled melts should be given extraordinary attention since the potential desires of SLN over nanoemulsions are related to the solid lipid state [59]. The main goal behind the production of supercooled melts is to focus on crystallization measures for size. Crystallization needs to begin with a simple number of cores for crystallization. This simple number of atoms is less likely to be framed in tiny droplets and the tendency to produce supercooled melts increases with decreasing droplet size along these lines [60].

In lipid dispersions, the range of super cooling will arrive at 30 to $40^{\circ} \mathrm{C}$. For eg, the melting temperature of Trilaurin is $>40^{\circ} \mathrm{C}$, but the lipid recrystallizes at temperatures below the freezing point of water in phospholipid-balanced Nano dispersions. Notwithstanding height, emulsifiers, inserted drugs and other factors may affect crystallization. In this way, the verification of the solid-state of the lipid by proper analytical techniques such as NMR, X-beam or DSC is necessary. Among these, NMR helps the presence of supercooled dissolves to be analysed incredibly rapidly and non-destructively [61].

\subsubsection{Lipid modifications}

The physical state of the lipid cannot be seen as crystallized or non-crystallized since the crystallized lipid can be achieved by a few crystal lattice modifications. In thermodynamically temperamental setups, lipid particles also established mobility. These arrangements thus have a lower density and, finally, a greater ability to incorporate guest molecules. The benefit of developed rates of integration of temperamental shifts is paid for by the drug's mobility. 
Throughout storage, for thermodynamically stable arrangements and sometimes correlated with drug particle removal, analysis of the crystal lattice may occur [62].

Although this boundary stimulates drug integration and drug release, the efficiency of the SLN mechanism will be determined to an enormous degree by lipid modulation. The validation of a device with characterized and consistent characteristics is desirable in terms of reproducibility and drug protection. Therefore, in unbalanced outlines, the tradition of the advanced drug loading edge requires the advancement of procedures to avoid alteration in storage. If this matter is followed up, the supplementary probabilities of altered opioid release profiles would be exposed. Jenning has occurred in vitro on the skin to demonstrate that the evaporation of water stimulates improvements in SLN dispersions that induce drug discharge from the lipid and result in increased drug penetration into the skin [63].

It stated that with the task of alpha, $\beta$, structure, the development of lipid variations is not generally assumed. Due to a few subspecies and lipid associations with the emulsifiers, randomness increases more. In addition, Westesen Bunch's late work indicates that the particle size itself is the deciding factor in SLN's physical properties. In the DSC thermogram, which corresponds to an alternative number of lipid layers of SLN particles, a few sharp peaks were observed [64].

\subsubsection{Particle shape}

The shape of nanoparticles of lipids can vary fundamentally from a sphere. In the platelet structure, lipids need to solidify. TEM has seen improvements in form amid Nanoemulsions and SLN. The shape of lipid crystals is not just a theoretical particle size issue [27]. Compared to spheres, platelet forms have a better portion of surface areas, so higher measurements of surfactants are needed for adjustment. Molecular sizes of $100 \mathrm{~nm}$ are converted into 20 tolerant lipid layers if the form is spherical. In any event, if there is a platelet structure, they convert to littler qualities [64]. Cryo transmission electron microscopy tests of nanoparticles of Trimyristin provide good evidence that PCS sizes are 130 $\mathrm{nm}$. A fraction of the advanced quantity of the substance would be legally confined to the particles outside, which conflicts with the general point of the SLN systems [65].

\subsubsection{Gelation phenomena}

It portrays the modification of the thick gel into a low-viscosity SLN dispersion. This sequence will happen unexpectedly and easily, too. As a rule, gel growth is an irreversible step that requires the loss of the size of the colloidal particle. Shear forces were also induced to be invigorated by the strong interaction of the SLN dispersion with various surfaces needle is a popular model. Results will be impacted by the production of objects if gelation occurs in vitro during preliminary SLN characterization steps. Nonetheless, the survival of this life form is put in danger if this happens during the i.v injection into living animal groups [66].

Siekmann suggested that the structure of gel is consistent with measurements of crystallization. Abnormal surfaces prompt the lipid crystals to be crystallized or altered or modified. Because of the chosen platelet structure (in $\beta$ alteration), this cycle is associated with an extension of the molecule surface. The molecules of the surfactant can no longer provide sufficient inclusion of new surfaces and particle aggregation is thus observed. The expansion of coemulsifying surfactants with accelerated mobility will retard or prevent gelation [67] [68].

In SLNs, high temperatures, light initiation and mechanical friction advance gelation. Particle development was stopped by preservation in darkness at $8^{\circ} \mathrm{C}$. Fat examples placed under nitrogen air were also shown to be more stable than experiments conducted under normal air [69].

Advance gelation with high lipid concentrations and high ionic qualities. A good predictor of gelation wonders has been the zeta potential. Stable cases had a zeta capability of $-25 \mathrm{mV}$, while the beginning of gelation phenomena was indicated by a zeta capability of $-15 \mathrm{mV}$. The steadiness of parenteral fat emulsions was accounted for by comparable qualities [70].

For the gelation approach, a few structures could be involved. Both gelation advertisers (high temperature, light, sheer pressure) increase the particles' kinetic energy and benefit the particles' effect. With temperature, the surfactant film can adjust its presentation. Additional views identify the crystallization energy and change between the transitions in lipids that would be precious by the components alluded to above. Fast lipid crystallization increases the duration of gelation [71].

Upon arrival of output, the crystallinity of Compritol SLN is almost high. During capacity, the liquid components crystallize. During a capacity season of 3 years, healthy SLN systems do not recrystallize and include a few lipid shifts. Examples of semi-solid contain only $\beta^{\prime}$ and $\beta^{\prime}$ modification, although total gelation prompts the change to $\beta^{\prime}$ alteration 
in full. In the stable structure, the presence of liquid phases progresses the crystallization because insecure crystals in the steady alteration will redissolve and solidify. Therefore, it is feasible to accelerate the transition in alpha- $\beta$ during RT potential without dissolving the Capitol [72]. By and wide, in the alpha shift, fatty compounds can take shape. Surfactants, for instance, poloxamer, will retard the alpha- $\beta$ modification. Freitas saw that there were comparative impacts on the nitrogen environment. Nitrogen retardation has been due to lipid hydrolysis inhibition [73][74]. Aggregates of each micron or less are the crystal scale of alpha modification of polycrystalline lipid totals. The modification discrepancy is related to molecule size expansion ( $\beta$ ': below $5 \mu \mathrm{m} ; \beta$ : $20-100 \mu \mathrm{m}$ ). TEM that Tristearin crystals also have a cubic form in the alpha shift of Tripalmitin. The modification of $\beta$ is generated from stapled spheroids and the stable modification of $\beta$ is generated from coagulated platelets since quite a while ago.

\section{In vitro method for the study of Drug release from Solid Lipid Nanoparticles}

Full SLN drug release studies identified by various scientists, Drug diffusion from SLNs dependent on particle size, Drug partition coefficient Slow drug release can be achieved after homogeneous distribution of the drug into the lipid matrix. Lipid carrier crystallization efficiency and fast drug movement contribute to rapid drug release, surfactant concentration and temperature both affect the profile of drug release [75]. In the drug release process, for example in the drug-enriched shell model, rapid early drug release (burst effect) obtained within minutes maintaining too broad surface area, the drug integration and processing methods of SLNs play a part. This was obtained because of drug accumulation on the surface of particles. With growing particle size, the burst release is decreased and extended release may be achieved if the particles were relatively large, i.e. lipid microparticles. The amount of surfactant also impacts the release of the prescription. The minimum quantity to a minimal blast and extended release of drugs of surfactant principals. In the case of a drug enriched core model, the drug is surrounded by a lipid layer whose release of drugs is a form regulated by the membrane and is directed by the diffusion of the Fick law [76].

It is possible to determine the in vitro profile using different techniques such as diffusion cell, regular dialysis, modified ultrafiltration method, etc. In vitro drug release from lipid nanoparticles such as phosphate buffer, using double chamber diffusion cells on a shaker stand, can be calculated as an improper dissolution medium in the receptor compartment, the dissolution medium is filled and the suspension of nanoparticulate is brought into the donor compartment. A dividing membrane is mounted between the two compartments. In order to assess the released drugusing normal protocol, the receptor compartment is observed by withdrawing a sufficient volume at various time intervals. The different methods have been used for in vitro release are Using diffusion cells with the artificial or biological membrane, Using dialysis bag, Reverse dialysis sac technique, Ultra-centrifugation, Centrifugal ultrafiltration technique [77].

\section{Uptake Mechanism of SLNs by Brain and In vivo Breakdown of Lipid to Release the Drug}

The drug can be diffused from lipid nanoparticles through diffusion and undergo further passage through the olfactory bulb and brain stem after intranasal administration of SLNs Figure 7, eventually accessing the brain or cerebrospinal fluid via the olfactory pathway. The additional pathway is a neuronal olfactory pathway that can be intraneuronal and extraneuronal [78]. The optimal nanoparticle dimensions for intranasal distribution are less than $100 \mathrm{~nm}$. SLNs can also be transported through the transcellular path via close junctions of epithelial cells to the degree that lamina propria is significantly less than paracellular, i.e. extraneuronal. Each or a mixture of these fates may be met as nanoparticles extend lamina propria: absorption into systemic circulation, absorption through deep cervical neck lymph nodes or extracellular diffusion through perineurial and perivascular spaces with eventual penetration into the cranial compartment. SLNs are made of biocompatible lipids that are physiologically related. The mechanism for transport and metabolism is also present in the body, which can largely subsidize the in vivo fate of the SLNs [79]. Lipases, which are found in different organs and tissues, may be the most important enzyme in SLN degradation. The ester linkage was broken by lipases and partial glycerides or glycerol and free fatty acids developed. Maximum lipases require an oil/water interface to trigger, activating the catalytic core. The lipase degradation of SLNs depends on their structure, i.e. the surfactant form and duration of fatty acid chains. Surfactants can speed up degradation due to steric stabilization (e.g. Cholic acid sodium salt) or hinder/slow (e.g. poloxamer 407, poloxamer 188, tween 80) [80]. 


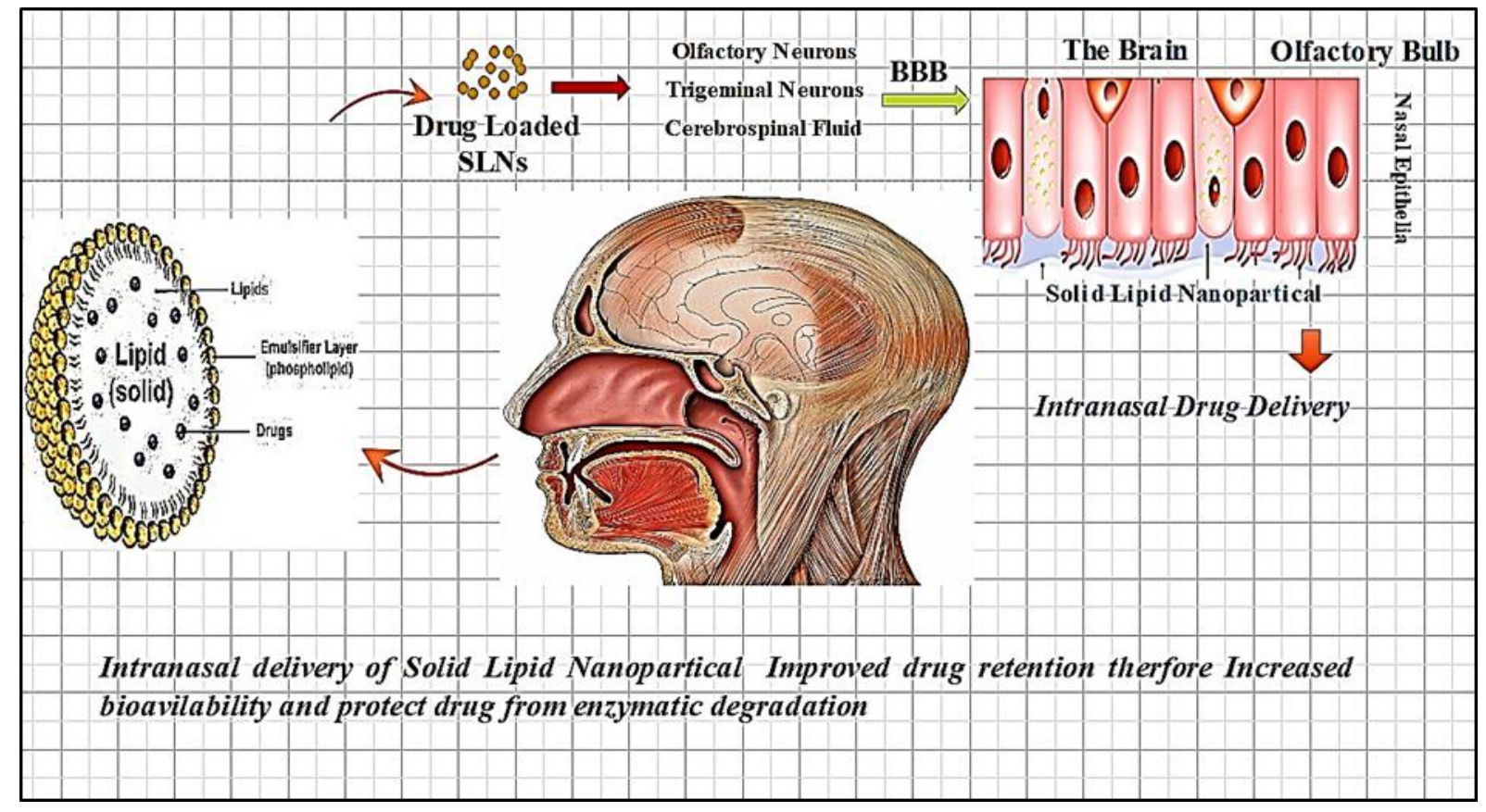

Figure 7 Uptake Mechanism of SLNs by Brain by crossing BBB

\section{Conclusion}

Solid lipid nanoparticles (SLNs) have developed as an outstanding nano colloidal system for drug delivery. SLNs have greatly picked up the attention of a few specialists with its outstanding properties and advantages over other Conventional dosage forms, and other colloidal partners of SLN have ended up being an enormous disclosure in nanotechnology due to their nominal performance and as a protected vehicle pharmaceutical drug delivery. SLN as a colloidal drug carrier places together the compensations of polymeric nanoparticles, SLNs have numerous benefits such as easy incorporation of lipid and lipophilic as well as hydrophilic drugs, suitable physical stability, and available at low cost and easy to manufacture. The nasal route was accepted to exploit first its prevention of the hepatic first-pass metabolism to increase the absolute bioavailability, and second, the direct nose-to-brain pathway to enhance the brain drug delivery. SLNs were designated as a drug delivery system to improve drug permeability across the blood-brain barrier (BBB) and consequently its brain delivery.

\section{Abbreviations}

SLNs - Solid Lipid Nanoparticles; HPH- High-Pressure Homogenization; BBB- Blood Brain Barrier; EE - Entrapment Efficiency; DL - Drug Loading; SEM- Scanning Electron Microscopy; TEM- Transmission Electron Microscopy; PSParticle Size; DSC- Differential Scanning Colorimetry; NMR- Nuclear Magnetic Radiations.

\section{Compliance with ethical standards}

\section{Acknowledgments}

Acknowledgments must be inserted here.

\section{Disclosure of conflict of interest}

If two or more authors have contributed in the manuscript, the conflict of interest statement must be inserted here.

\section{References}

[1] JE Hulla, SC Sahu, AW Hayes. Nanotechnology: History and future, Journals.Sagepub.Com. 2015; 34: 1318-1321.

[2] C Vauthier, K Bouchemal, Expert Review Methods for the Preparation and Manufacture of Polymeric Nanoparticles, Springer. 2009; 26: 1025-1058. 
[3] A.S.-I. journal of pharmaceutics, undefined 1986, Evaluation of poly (lactic acid) as a biodegradable drug delivery system for parenteral administration, Elsevier. (n.d.).

[4] D Zhang, W Dai, C Duan, L Jia, Y Wang, F Feng, Q Zhang. Preparation and characteristics of oridonin-loaded nanostructured lipid carriers as a controlled-release delivery system, J. Microencapsul. 2010; 27: 234-241.

[5] A. Beloqui, M. Solinís, A.R.-G.- B. Medicine, undefined 2016, Nanostructured lipid carriers: promising drug delivery systems for future clinics, Elsevier. (n.d.).

[6] Y. Luo, D. Chen, L. Ren, X. Zhao, J. Qin. Solid lipid nanoparticles for enhancing vinpocetine's oral bioavailability, Elsevier. 2006.

[7] A. Silva, E. González-Mira, M. García, M.E.-C., S.B. undefined 2011, Preparation, characterization and biocompatibility studies on risperidone-loaded solid lipid nanoparticles (SLN): high pressure homogenization versus ultrasound, Elsevier. (n.d.).

[8] C Schwarz, W Mehnert, J Lucks, RM.-J. of controlled release, undefined 1994, Solid lipid nanoparticles (SLN) for controlled drug delivery. I. Production, characterization and sterilization, Elsevier. (n.d.).

[9] A zur Mühlen, C Schwarz, W.M.-E. journal of pharmaceutics, undefined 1998, Solid lipid nanoparticles (SLN) for controlled drug delivery-drug release and release mechanism, Elsevier. (n.d.).

[10] A. Garud, D. Singh, N. Garud. Solid Lipid Nanoparticles (SLN): Method, Characterization and Applications. 2012.

[11] DZ Hou, CS Xie, KJ Huang, CH Zhu. The production and characteristics of solid lipid nanoparticles (SLNs), Biomaterials. 2003; 24: 1781-1785.

[12] T Hai, X Wan, DG Yu, K Wang, Y Yang, ZP Liu. Electrospun lipid-coated medicated nanocomposites for an improved drug sustained-release profile, Mater. Des. 2016; 162: 70-79.

[13] R Müller, A Dingler, T.S.-H. of, undefined 2000, Large scale production of solid lipid nanoparticles (SLN ${ }^{\mathrm{TM}}$ ) and nanosuspensions (DissoCubes ${ }^{\mathrm{TM}}$ ), Books.Google.Com. (n.d.).

[14] J Liu, J Zhu, Z Du, B Qin. Preparation and pharmacokinetic evaluation of Tashinone IIA solid lipid nanoparticles, Drug Dev. Ind. Pharm. 2005; 31: 551-556.

[15] S Weber, A Zimmer, J Pardeike. Solid Lipid Nanoparticles (SLN) and Nanostructured Lipid Carriers (NLC) for pulmonary application: A review of the state of the art, Eur. J. Pharm. Biopharm. 2014; 86: 7-22.

[16] K. Bhaskar, C. Krishna Mohan, M. Lingam, V. Prabhakar Reddy, V. Venkateswarlu, Y. Madhusudan Rao. Development of nitrendipine controlled release formulations based on SLN and NLC for topical delivery: In vitro and ex vivo characterization, Drug Dev. Ind. Pharm. 2008; 34: 719-725.

[17] A Garcês, MH Amaral, JM Sousa Lobo, AC Silva. Formulations based on solid lipid nanoparticles (SLN) and nanostructured lipid carriers (NLC) for cutaneous use: A review, Eur. J. Pharm. Sci. 2018; 112: 159-167.

[18] V Lingayat, N Zarekar, R.S.-N. undefined, Solid lipid nanoparticles: a review, Academia.Edu. (n.d.). 2017.

[19] R. Article, M.K. Sarangi, S. Padhi. SOLID LIPID NANOPARTICLES-A REVIEW, 2016.

[20] S. Kumar, M. Kumar Patel, Sandeep Hanumanaik, K. Ramya Sree. SOLID LIPID NANOPARTICLES; A REVIEW Periodontal treatment View project Application of Nanotechnology in environment and medical View project SOLID LIPID NANOPARTICLES; A REVIEW, Artic. Int. J. Pharm. Sci. Res. 2013; 4: 928-940.

[21] P Blasi, S Giovagnoli, A Schoubben. M.R.-A. drug delivery, undefined 2007, Solid lipid nanoparticles for targeted brain drug delivery, Elsevier. (n.d.). 2020.

[22] P Kaur, K.K.-I. journal of pharmaceutics, undefined 2008, Pharmacokinetics and brain uptake of diazepam after intravenous and intranasal administration in rats and rabbits, Elsevier. (n.d.). 2020.

[23] C Pardeshi, P Rajput, V Belgamwar, AT. Pharmaceutica, undefined, Solid lipid based nanocarriers: an overview, Hrcak.Srce.Hr. (n.d.). 2012.

[24] R Müller, W Mehnert, J.L.-E. journal of, undefined 1995, Solid lipid nanoparticles (SLN): an alternative colloidal carrier system for controlled drug delivery, Pascal-Francis.Inist.Fr. (n.d.). 2020.

[25] P.B.-C.R. in T.D. Carrier, undefined 2004, Physical chemical considerations of lipid-based oral drug deliverysolid lipid nanoparticles, Dl.Begellhouse.Com. (n.d.). 2020. 
[26] M Joshi, S Pathak, S Sharma, V.P.-I. journal of, undefined 2008, Design and in vivo pharmacodynamic evaluation of nanostructured lipid carriers for parenteral delivery of artemether: Nanoject, Elsevier. (n.d.). 2008.

[27] B. Siekmann, K.W.-P.P. Lett. undefined 1992, Submicron-sized parenteral carrier systems based on solid lipids, (n.d.). 1992.

[28] D Crommelin, HSK J, M Dekker, N York. undefined 1994, Colloidal drug delivery systems, (n.d.). 1994.

[29] S Patel, M Patel, A Patel, M.C.-N.-B, U. Solid lipid nPatel, S., Patel, M., Patel, A., ... M.C.-N.-B., 2018, undefined, n.d. Solid lipid nanoparticles for targeted brain drug delivery. Elsevier.anoparticles for targeted brain drug delivery, Elsevier. (n.d.). 2018.

[30] R. Müller, K.P.-I. journal of pharmaceutics, undefined 1998, Nanosuspensions for the formulation of poorly soluble drugs: I. Preparation by a size-reduction technique, Elsevier. (n.d.). 1998.

[31] DJ McClements, J Rao. Food-Grade nanoemulsions: Formulation, fabrication, properties, performance, Biological fate, and Potential Toxicity, Crit. Rev. Food Sci. Nutr. 2011; 51: 285-330.

[32] A Zur Mühlen, WM- Pharmazie. undefined 1998, Drug release and release mechanism of prednisolone loaded solid lipid nanoparticles, Pascal-Francis.Inist.Fr. (n.d.). 1998.

[33] SJ Hur, EA Decker, DJ McClements. Influence of initial emulsifier type on microstructural changes occurring in emulsified lipids during in vitro digestion, Food Chem. 2009; 114: 253-262.

[34] R Cavalli, E Peira, O Caputo, M.G.-I. journal of, undefined 1999, Solid lipid nanoparticles as carriers of hydrocortisone and progesterone complexes with $\beta$-cyclodextrins, Elsevier. (n.d.).

[35] N Naseri, H Valizadeh, P Zakeri-Milani. Solid lipid nanoparticles and nanostructured lipid carriers: Structure preparation and application, Adv. Pharm. Bull. 2015; 5: 305-313.

[36] M Durán-Lobato, A Enguix-González, M Fernández-Arévalo, L Martín-Banderas. Statistical analysis of solid lipid nanoparticles produced by high-pressure homogenization: A practical prediction approach, J. Nanoparticle Res. 2013; 15: 1-14.

[37] S Patel, M Patel, A Patel, M.C.-N.-B. undefined 2018, Solid lipid nanoparticles for targeted brain drug delivery, Elsevier. (n.d.). 2018.

[38] V Jenning, A Lippacher. S.G.-J. of microencapsulation, undefined 2002, Medium scale production of solid lipid nanoparticles (SLN) by high pressure homogenization, Taylor Fr. (n.d.). 2002.

[39] V Yadav, S AlokMahor, S Alok, A.A.-W.J.P. undefined 2014, Solid lipid nanoparticles (sln): formulation by high pressure homogenization, Academia.Edu. (n.d.). 2014.

[40] A. Dingler, S.G.-J. of microencapsulation, undefined 2002, Production of solid lipid nanoparticles (SLN): scaling up feasibilities, Taylor Fr. (n.d.).

[41] EB Souto, S Doktorovova, A Zielinska, AM Silva. Key production parameters for the development of solid lipid nanoparticles by high shear homogenization, Pharm. Dev. Technol. 2019; 24: 1181-1185.

[42] V Jenning, AF Thünemann, SH Gohla, Characterisation of a novel solid lipid nanoparticle carrier system based on binary mixtures of liquid and solid lipids, Int. J. Pharm. 2000; 199: 167-177.

[43] M.G.-P.T. Europe, undefined 1997, Solid Lipid Nanospheres from Warm Micro-Emulsions: Improvements in SLN production for more efficient drug delivery, AVANSTAR Commun. (n.d.). 1977.

[44] L Anurak, G Chansiri, D Peankit, K Somlak. Griseofulvin solid lipid nanoparticles based on microemulsion technique, in: Adv. Mater. Res., Trans Tech Publications Ltd. 2011; 47-50.

[45] D Quintanar-Guerrero, D Tamayo-Esquivel, A Ganem-Quintanar, E Allémann, E Doelker. Adaptation and optimization of the emulsification-diffusion technique to prepare lipidic nanospheres, Eur. J. Pharm. Sci. 2005; 26: 211-218.

[46] JQ Zhang, J Liu, XL Li, BR Jasti. Preparation and Characterization of Solid Lipid Nanoparticles Containing Silibinin, Drug Deliv. 2007; 14: 381-387.

[47] B Sjöström, BB.-I. journal of pharmaceutics, undefined 1992, Preparation of submicron drug particles in lecithinstabilized o/w emulsions I. Model studies of the precipitation of cholesteryl acetate, Elsevier. (n.d.). 1992.

[48] M Trotta, F Debernardi, O.C.-I. journal of pharmaceutics, undefined 2003, Preparation of solid lipid nanoparticles by a solvent emulsification-diffusion technique, Elsevier. (n.d.). 2003. 
[49] A. de Labouret, O Thioune, H Fessi, JP Devissaguet, F Puisieux. Application of an original process for obtaining colloidal dispersions of some coating polymers. Preparation, characterization, industrial scale-up, Drug Dev. Ind. Pharm. 1995; 21: 229-241.

[50] H Heiati, R Tawashi, NC Phillips, Drug retention and stability of solid lipid nanoparticles containing azidothymidine palmitate after autoclaving, storage and lyophilization, J. Microencapsul. 1998; 15: 173-184.

[51] M Pikal, S Shah, M Roy, R.P.-I. journal of, undefined 1990, The secondary drying stage of freeze drying: drying kinetics as a function of temperature and chamber pressure, Elsevier. (n.d.). 1990.

[52] H. Hauser, G.S.-B.A. of Lipid, undefined 1988, Stabilization of small, unilamellar phospholipid vesicles by sucrose during freezing and dehydration, Springer. (n.d.). 1988.

[53] S Vemuri, C Der Yu, JS Degroot, V Wangsatornthnakun, S Venkataram. Effect of sugars on freeze-thaw and lyophilization of liposomes, Drug Dev. Ind. Pharm. 1991; 17: 327-348.

[54] R Lander, W Manger, M Scouloudis, A Ku, C Davis, A Lee, Gaulin Homogenization: A Mechanistic Study, Wiley Online Libr. 2000; 16: 80-85.

[55] H.W.-U. Berlin D. of Pharmacy, undefined Berlin, undefined 1995, Feste lipid-nanopartikel (sln) für die gewebsspezifishe arzneistoffapplikation, (n.d.). 1995.

[56] B. Wei, C. Cai, Z. Jin, Y. Tian, High-pressure homogenization induced degradation of amylopectin in a gelatinized state, Starch - Stärke. 2016; 68: 734-741.

[57] B Wei, C Cai, B Xu, Z Jin, Y Tian. Disruption and molecule degradation of waxy maize starch granules during high pressure homogenization process, Food Chem. 2018; 240: 165-173.

[58] RH Müller, SA Runge, V Ravelli, AF Thünemann, W Mehnert, EB Souto. Cyclosporine-loaded solid lipid nanoparticles (SLN@): Drug-lipid physicochemical interactions and characterization of drug incorporation, Eur. J. Pharm. Biopharm. 2008; 68: 535-544.

[59] A Saupe, KC Gordon, T Rades. Structural investigations on nanoemulsions, solid lipid nanoparticles and nanostructured lipid carriers by cryo-field emission scanning electron microscopy and Raman spectroscopy, Int. J. Pharm. 2006; 314: 56-62.

[60] N Garti, K Sato. Crystallization and polymorphism of fats and fatty acids. 1988.

[61] K Westesen, H.B.-I. journal of pharmaceutics, undefined 1995, Do nanoparticles prepared from lipids solid at room temperature always possess a solid lipid matrix?, Elsevier. (n.d.). 1995.

[62] A Puri, K Loomis, B Smith, JH Lee, A Yavlovich, E Heldman, R Blumenthal. Lipid-based nanoparticles as pharmaceutical drug carriers: From concepts to clinic, Crit. Rev. Ther. Drug Carrier Syst. 2009; 26: 523-580.

[63] V Jenning, M Schäfer-Korting. S.G.-J. of controlled release, undefined 2000, Vitamin A-loaded solid lipid nanoparticles for topical use: drug release properties, Elsevier. (n.d.). 2000.

[64] T. Unruh, H. Bunjes, K. Westesen, M.H.J. Koch. Observation of size-dependent melting in lipid nanoparticles, J. Phys. Chem. B. 1999; 103: 10373-10377.

[65] B Siekmann, K.W.-E.J. of P. Sciences, undefined 1994, P287 electron-microscopic characterization of melthomogenized solid lipid nanoparticles, Elsevier. (n.d.). 1994.

[66] K Westesen, B Siekmann. Investigation of the gel formation of phospholipid-stabilized solid lipid nanoparticles, Int. J. Pharm. 1997; 151: 35-45.

[67] B Siekmann, K.W.-C. and surfaces B. Biointerfaces, undefined 1994, Thermoanalysis of the recrystallization process of melt-homogenized glyceride nanoparticles, Elsevier. (n.d.). 1994.

[68] K Westesen, B.S.-I. journal of pharmaceutics, undefined 1997, Investigation of the gel formation of phospholipidstabilized solid lipid nanoparticles, Elsevier. (n.d.). 1997.

[69] C Freitas, RH Müller. Stability determination of solid lipid nanoparticles (SLN®) in aqueous dispersion after addition of electrolyte, J. Microencapsul. 1999; 16: 59-71.

[70] C Freitas, R.M.-I. journal of pharmaceutics, undefined 1998, Effect of light and temperature on zeta potential and physical stability in solid lipid nanoparticle (SLNTM) dispersions, Elsevier. (n.d.). 1998.

[71] H Bunjes, K Westesen. M.K.-I. journal of pharmaceutics, undefined 1996, Crystallization tendency and polymorphic transitions in triglyceride nanoparticles, Elsevier. (n.d.). 1996. 
[72] C Freitas, R.M.-E. journal of pharmaceutics and, undefined 1999, Correlation between long-term stability of solid lipid nanoparticles (SLN ${ }^{\mathrm{TM}}$ ) and crystallinity of the lipid phase, Elsevier. (n.d.). 1999.

[73] H YOSHINO, M.K.-C. and undefined 1983, NII-Electronic Library Service, n.d. 1983.

[74] N.G.-C. and polymorphism of fats and fatty, undefined 1988, Effects of surfactants on crystallization and polymorphic transformation of fats and fatty acids, Marcel Dekker, New York. (n.d.). 1988.

[75] V Venkateswarlu, K Manjunath. Preparation, characterization and in vitro release kinetics of clozapine solid lipid nanoparticles, J. Control. Release. 2004; 95: 627-638.

[76] M Yasir, UVS Sara. Solid lipid nanoparticles for nose to brain delivery of haloperidol: In vitro drug release and pharmacokinetics evaluation, Acta Pharm. Sin. B. 2014; 4: 454-463.

[77] B Magenheim, MY Levy, S Benita. A new in vitro technique for the evaluation of drug release profile from colloidal carriers - ultrafiltration technique at low pressure, Int. J. Pharm. 1993; 94: 115-123.

[78] S Martins, S Costa-Lima, T Carneiro, A Cordeiro-Da-Silva, EB Souto, DC Ferreira. Solid lipid nanoparticles as intracellular drug transporters: An investigation of the uptake mechanism and pathway, Int. J. Pharm. 2012; 430: 216-227.

[79] Y Gupta, A Jain, SK Jain. Transferrin-conjugated solid lipid nanoparticles for enhanced delivery of quinine dihydrochloride to the brain, J. Pharm. Pharmacol. 2007; 59: 935-940.

[80] S Sadegh Malvajerd, A Azadi, Z Izadi, M Kurd, T Dara, M Dibaei, M Sharif Zadeh, H Akbari Javar, M Hamidi. Brain Delivery of Curcumin Using Solid Lipid Nanoparticles and Nanostructured Lipid Carriers: Preparation, Optimization, and Pharmacokinetic Evaluation, ACS Chem. Neurosci. 2019; 10: 728-739. 